\title{
Translational Research in Stroke
}

Cerebrovascular Diseases
Cerebrovasc Dis 2020;49:334-340

DOI: $10.1159 / 000508578$
Received: March 5, 2020

Accepted: May 6, 2020

Published online: June 24, 2020

\section{Aeromedical Retrieval for Stroke in Australia}

\author{
Fergus William Gardiner ${ }^{a, b}$ Lara Bishop ${ }^{a, b}$ Angela Dos Santos ${ }^{d, e, f}$ \\ Pritish Sharma $^{a}$ Damien Easton ${ }^{d, e, f}$ Frank Quinlan ${ }^{a}$ Leonid Churilov ${ }^{c, f}$ \\ Madeleine Schwarz $^{g}$ Silke Walter ${ }^{g}$ Klaus Fassbender ${ }^{g}$ Stephen M. Davis ${ }^{d, e, f}$
}

Geoffrey A. Donnand, $e, f$

aThe Royal Flying Doctor Service, Federation Office, Barton, ACT, Australia; ${ }^{\text {b} N a t i o n a l ~ C e n t r e ~ f o r ~ E p i d e m i o l o g y ~}$ and Population Health, Research School of Population Health, The Australian National University, Canberra, ACT, Australia; 'Department of Medicine (Austin Health), The University of Melbourne, Melbourne, VIC, Australia; ${ }^{d}$ Royal Melbourne Hospital, Parkville, Melbourne, VIC, Australia; ${ }^{e}$ Faculty of Medicine, Dentistry and Health Sciences, University of Melbourne, Melbourne, VIC, Australia; ${ }^{\mathrm{N}}$ Melbourne Brain Centre at the Royal Melbourne Hospital, The University of Melbourne, Melbourne, VIC, Australia; ${ }^{9}$ Department of Neurology, University Hospital of the Saarland, Homburg/Saar, Germany

\section{Keywords}

Stroke · Aeromedical · Rural · Accessibility · Equity ·

Indigenous

\begin{abstract}
Introduction: Rural, remote, and Indigenous stroke patients have worse stroke outcomes than urban Australians. This may be due to lack of timely access to expert facilities. Objectives: We aimed to describe the characteristics of patients who underwent aeromedical retrieval for stroke, estimate transfer times, and investigate if flight paths corresponded with the locations of stroke units (SUs) throughout Australia. Methods: Prospective review of routinely collected Royal Flying Doctor Service (RFDS) data. Patients who underwent an RFDS aeromedical retrieval for stroke, July 2014-June 2018 (ICD-10 codes: 160-I69), were included. To define the locations of SUs throughout Australia, we accessed data from the 2017 National Stroke Audit. The main outcome measures included determining the characteristics of patients with an in-
\end{abstract}

flight diagnosis of stroke, their subsequent pickup and transfer locations, and corresponding SU and imaging capacity. Results: The RFDS conducted 1,773 stroke aeromedical retrievals, consisting of 1,028 (58\%) male and 1,481 (83.5\%) non-Indigenous and 292 (16.5\%) Indigenous patients. Indigenous patients were a decade younger, 56.0 (interquartile range [IQR] 45.0-64.0), than non-Indigenous patients, 66.0 (IQR 54.0-76.0). The most common diagnosis was "stroke not specified," reflecting retrieval locations without imaging capability. The estimated median time for aeromedical retrieval was 238 min (95\% confidence interval: 231-244). Patients were more likely to be transferred to an area with SU and imaging capability (both $p<0.0001$ ). Conclusion: Stroke patients living in rural areas were younger than those living in major cities (75 years, Stroke Audit Data), with aeromedically retrieved Indigenous patients being a decade younger than non-Indigenous patients. The current transfer times are largely outside the time windows for reperfusion methods. Future research should aim to facilitate more timely diagnosis and treatment of stroke.

(c) 2020 S. Karger AG, Basel

\section{KARGER}

(c) 2020 S. Karger AG, Basel

karger@karger.com

www.karger.com/ced
Fergus William Gardiner

Royal Flying Doctor Service

Federation Office, Level 2

1012 Brisbane Avenue, Barton, ACT 2600 (Australia)

fergus.gardiner@rfds.org.au 


\section{Introduction}

Stroke is a massive global health challenge. With 13.5 million new strokes each year and 5.5 million deaths, stroke is a leading cause of mortality and chronic disability [1]. The most effective intervention for acute stroke, apart from thrombolysis or endovascular clot retrieval, is management in a specialised stroke unit (SU) [1]. Importantly, the majority of these reperfusion therapies are best administered within $4.5 \mathrm{~h}$ of stroke onset. Times beyond this, sometimes up to $24 \mathrm{~h}$, may only be considered when more sophisticated imaging techniques are applied to detect the presence of viable brain tissue, the ischaemic penumbra [2]. Most Australians living in rural and remote areas do not have access to SUs [3], with the majority required to travel more than $200 \mathrm{~km}$ by road to access medical care [4]. As such, many patients living in rural and remote areas require aeromedical retrieval by the Royal Flying Doctor Service (RFDS).

However, it is unclear how many stroke patients undergo RFDS aeromedical retrievals, from remote and very remote Australia, to inner regional areas and major city hospitals. Their demographic characteristics and stroke subtypes are also unknown. Furthermore, the time sequence of events from stroke onset to eventual arrival at a healthcare facility capable of diagnosis and treatment is unclear.

Hence, we aimed to provide the first information on the characteristics of patients, who underwent an RFDS aeromedical retrieval for stroke during a set period throughout Australia. We aimed to estimate the times between first notification and arrival to the treating hospital airstrip and to test the hypothesis that receiving and transfer locations have different SU and imaging capability.

\section{Methods}

\section{Setting}

Australia is a vast continent, spanning 7.692 million square kilometres, making it the 6th largest country by total area [5]. The population of Australia in 2018 was $24,992,860$, with the majority of people residing in major city areas $(n=18,003,544 ; 72.0 \%)$ or inner-regional areas $(n=4,445,356 ; 17.8 \%)$, with the remainder in outer-regional $(n=2,052,366 ; 8.2 \%)$, remote $(n=291,213 ; 1.2 \%)$, and very remote $(n=200,381 ; 0.1 \%)$ areas [6]. Indigenous Australians accounted for a substantial proportion of the remote $(n=$ $53,000 ; 18.2 \%)$ and very remote $(n=94,579 ; 47.2 \%)$ population. Australia has a relatively small population given its land mass, with the majority of people living in rural and remote areas distributed over vast distances, with poor access to permanent health services as a result of the "tyranny of distance" [7].

Aeromedical Retrieval for Stroke in Australia
Due, in part, to the tyranny of distance, the RFDS provides essential aeromedical and primary healthcare to rural and remote populations who are unable to access traditional services through the Medicare Benefits Schedule. The terms "remote" and "very remote" reflected areas classified as remote or very remote (RA4 and RA5, respectively) by the Australian Statistical Geography Standard (ASGS) [6].

RFDS aeromedical retrievals include primary evacuations (PEs) and inter-hospital transfers (IHTs). A PE describes the provision of emergency medical services to people affected by illness or accident, who are in a serious or potentially life-threatening condition, and who are beyond the normal medical infrastructure. An IHT describes the transfer of patients between hospitals designated as normal medical infrastructure, often to access a higher level of care, such as specialist treatment or life-saving surgery. However, an IHT can also include the transfer of a patient from a larger to a smaller community hospital, to receive ongoing care closer to the patient's home.

During stroke aeromedical retrieval, the RFDS crew stabilise the patient. In the majority of cases, baseline blood work, brain computerised tomography (CT) scanning, neurological consultation, and, when appropriate, tissue plasminogen activator (tPA) administration, are done at the receiving hospital. This is due to a lack of sophisticated services, including tertiary hospitals, in the majority of rural and remote retrieval locations [8].

\section{Patients}

All patients categorised as having an in-flight working diagnosis of stroke (ICD-10 codes: I60-I69), who had an aeromedical retrieval between July 1, 2014, and June 30, 2018, were included. Additional data collected within flight included the patient's sex, age, and self-reported Indigenous status, using either paper-based or electronic methods, according to the usual practice of the specific RFDS Section and Operation [9].

\section{Flight Data}

RFDS clinical and aeromedical databases were accessed to provide flight information, including the number of patient flight legs and flight time metrics. Specifically, we collected the following time metric components: time and date of initial call to RFDS, flight authorisation, flight mobilisation, arrival at scene or healthcare facility, departure from scene/health facility, and arrival at destination healthcare facility airstrip.

To inform us about the relevance of the destination healthcare facilities, we used SU location data from the most recent National Stroke Audit, which is conducted regularly by the Stroke Foundation [10]. These data included SU subtypes such as those with capacity to conduct thrombolysis and/or endovascular clot retrieval. Further, to determine the imaging capability of destination and receiving areas, we used data from the Australian Bureau of Statistics (ABS) to derive 2018 geographical population estimates. We defined "radiology/imaging services," as registered on Health Direct. We then used the RFDS Service Planning and Operational Tool (SPOT) to map coverage of radiology/imaging services across Australia, with a focus on remote and very remote Australia. Working from a geographic distribution of "demand (population = aeromedical stroke patients)" and a set of healthcare facilities that provide cover for a range of services (in this case, radiology/imaging services), SPOT calculates the proportion of demand covered by those facilities within a user-specified 


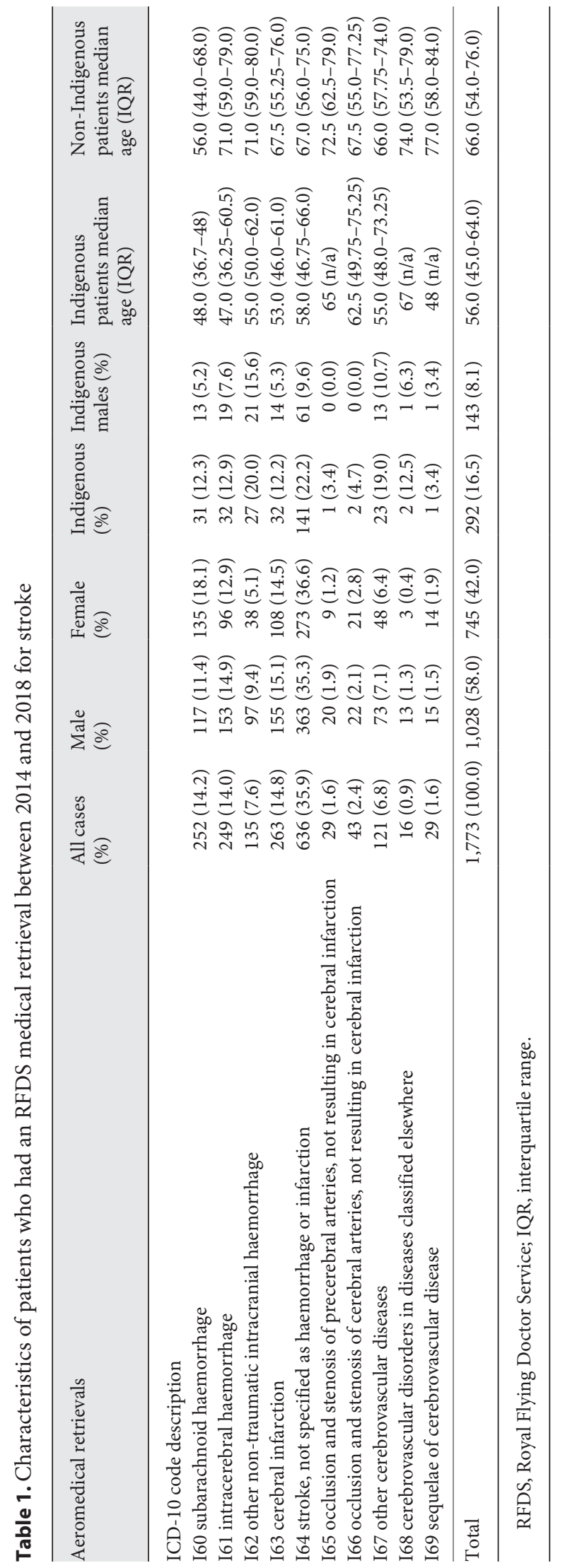

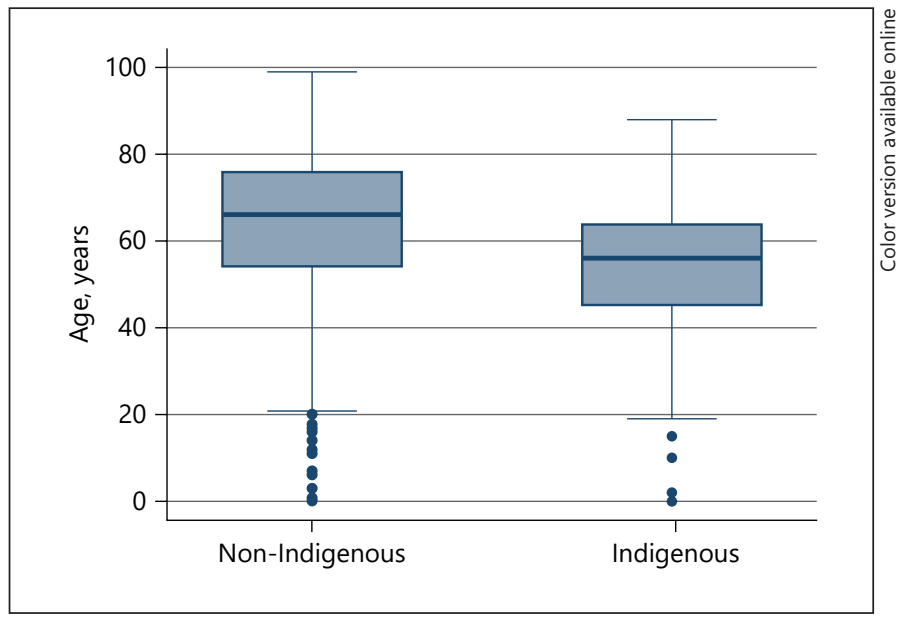

Fig. 1. Patient age distribution by Indigenous status. This figure demonstrates that there were significant age differences between non-Indigenous and Indigenous patient aeromedical transfers for stroke within rural and remote Australia.

drive time [4]. Demand is represented by population levels in different categories (e.g., radiology/imaging services).

\section{Statistical Analysis}

Continuous variables were summarised as means and standard deviations or medians and interquartile ranges (IQRs) based on the nature of the underlying distribution. Categorical variables were summarised as counts and proportions. Age differences were investigated using the Wilcoxon-Mann-Whitney test. The median, 25th, and 75th percentiles of transfer times distributions and respective $95 \%$ confidence intervals (95\% CIs) were estimated by bootstrapping individual flight data time metric components with 1,000 replications. The association between the location (transfer vs. receiving) and presence of SU or imaging capability was investigated using McNemar's test. $p$ values of $<0.05$ were regarded as indicative of statistical significance. Statistical analyses were performed using the statistical software package $\mathrm{R}$ version 3.5.1. Geographic mapping was conducted using Tableau Mapping Software [11].

\section{Results}

During the study period July 2014-June 2018 financial years, the RFDS conducted 105,147 aeromedical retrievals, of which 1,773 (1.7\%, 95\% CI: 1.6-1.8\%) fulfilled the criteria for stroke, as detailed in Table 1 . This equated to 8.5 retrievals for stroke per week, averaging just over 1 per day.

Of the 1,773 patients retrieved for suspected stroke, $58.0 \%$ were male, $83.5 \%(n=1,481)$ non-Indigenous, and $16.5 \%$ Indigenous. The median age was 64.0 years (IQR $52.0-75.0)$, with non-significant age $(p>0.05)$ differenc- 


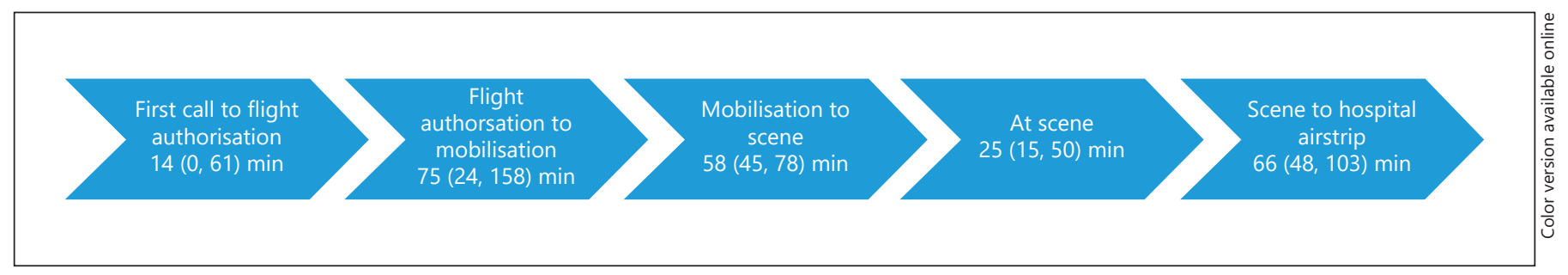

Fig. 2. Time metrics for key process components (median (interquartile range)), for RFDS aeromedical retrievals for stroke, July 1, 2014, to June 30, 2018. This figure details the time that a patient aeromedical transfer takes, from first call to delivery at the receiving hospital.

es between male and female patients (males 64.0, IQR 53.0-75.0; females 64.0, IQR 51-75.0). The average age of Indigenous patients was significantly $(p<0.05)$ younger at 56.0 (IQR 45.0-64.0) years, a decade younger than that of the non-Indigenous patients (average age of non-Indigenous was 66.0, IQR 54.0-76.0). See Table 1 for distribution by diagnosis and Figure 1 for distribution by age range and Indigenous status.

The most common stroke diagnosis was ICD code I64, "Stroke not specified as haemorrhage or infarction," at $35.9 \%(n=636)$. Of stroke retrievals with a more specific diagnosis, intracranial haemorrhage (14.0\%) and cerebral infarction $(14.8 \%)$ were the most common. There were significant differences in stroke diagnoses between gender groups. Males were more likely than females to have a diagnosis of intracerebral haemorrhage, other nontraumatic intracranial haemorrhage, cerebral infarction, and other cerebrovascular diseases. Conversely, females were more likely to have a diagnosis of subarachnoid haemorrhage and stroke not specified as haemorrhage or infarction.

Data on the kilometres flown were collected for 1,134 of $1,773(64.0 \%)$ patients. The median flight distance was $290.8 \mathrm{~km}$ (IQR $211 \mathrm{~km}, 500 \mathrm{~km}$ ). The estimated median transfer time was $238 \mathrm{~min}$ (95\% CI: 231-244) or $4 \mathrm{~h}$, from the time the RFDS received the first call to patient delivery at a hospital airstrip via an aeromedical retrieval (Fig. 2). Twenty-five percent of patients were transferred within $130 \min$ (95\% CI: $125-136)$ and $75 \%$ of patients within 450 min (95\% CI: 432-474).

The majority of SUs within Australia are located in inner-regional areas or major cities, as detailed in Figure 3. Of patients transferred from an area without an SU $(n=1,302), 1,106(85.0 \%)$ were transferred to an area with an SU. Of the patients transferred from an area with an SU $(n=470), 405(86.0 \%)$ were transferred to another more comprehensive SU. Patients were significantly $(p<$ $0.0001)$ more likely to be transferred from an area without an SU to an area with an SU. Of patients transferred from an area without imaging $(n=893), 876(98.0 \%)$ were transferred to an area with imaging. Of the patients transferred from an area with imaging $(n=879), 869(99.0 \%)$ were transferred to another area with imaging. Patients were significantly $(p<0.0001)$ more likely to be transferred from an area without imaging to an area with imaging.

\section{Discussion}

We have provided the first information on the retrievals by air for patients with a diagnosis of stroke in Australia. There were several important findings, which may have implications for future healthcare planning. First, the absolute numbers of patients were high at 1,773, a somewhat greater proportion being males and representing $1.7 \%$ of air retrievals during this period. Second, the proportion of patients who were Indigenous was high at $16.5 \%$, significantly greater than the average for Australia as a whole (3.0\%). Indigenous patients were also much younger. Third, the majority of patients did not have a specific diagnostic subtype of stroke established, particularly intracranial haemorrhage versus infarction, thus making early therapeuticintervention impossible. Fourth, time delays of transfer were such that even in cases where a specific diagnosis was made, the therapeutic time windows were beyond those for standard thrombolysis without more sophisticated imaging assessment. Finally, many of the transfers were made to centres with designated SUs and appropriate imaging facilities.

The median age of our cohort was 62.7 years. The younger age of Indigenous patients compared with nonIndigenous patients is striking and consistent with other Australian hospital-based studies. For example, a recent study of stroke patients seen at the Royal Adelaide Hospital found that the average age was much lower in Ab- 


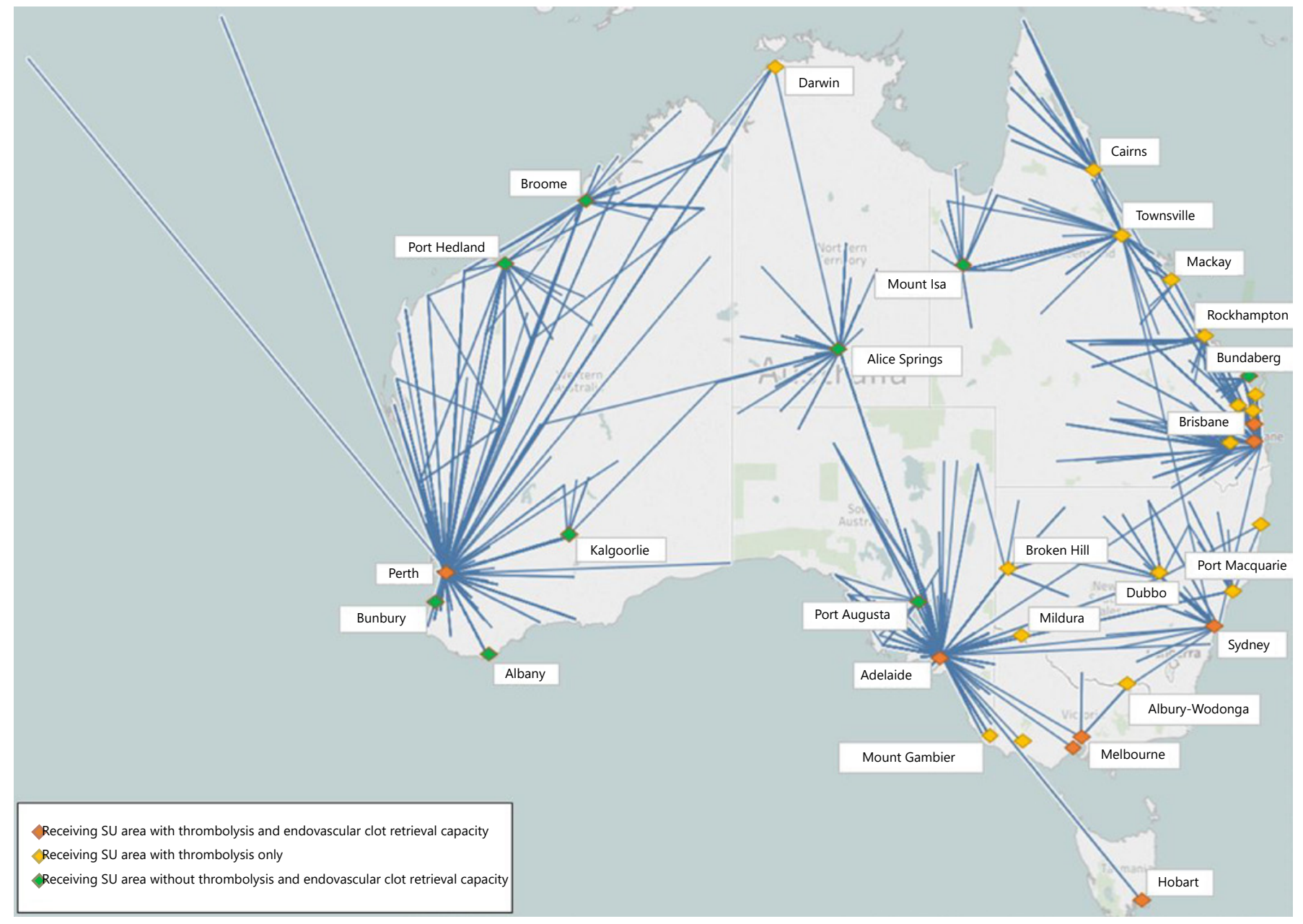

Fig. 3. Australian aeromedical retrievals for stroke and receiving Stroke Unit locations, July 1, 2014, to June 30, 2018. This figure details the aeromedical retrieval pick-up and transfer locations (detailed as a blue spider map), and the receiving areas Stroke Unit capacity (detailed as orange, yellow, and green diamonds).

original than in non-Aboriginal people (51 vs. 78 years, $p<0.001)$ and that the age-standardised stroke incidence per 100,000 in Aboriginal people was nearly double that in non-Aboriginal people $(p<0.001)$ [12]. The cause of this disparity is unclear; however, it may in part be linked to the higher rates of cardiovascular risk factors, coupled with discrimination and disadvantage (including economic, environmental, and educational), institutional racism, communication barriers and the lack of culturally appropriate services, and transportation to health centres in rural and remote Australia [13].

The estimated median time from first notification to patient arrival at hospital was $238 \mathrm{~min}(4 \mathrm{~h})$. These figures are higher than the Australia metropolitan average of 150 min [14], and services using the Prehospital Acute Stroke Triage (PAST) protocol of $90.5 \mathrm{~min}$ from symptom onset to emergency department arrival [15]. As mentioned, the majority of aeromedical retrievals came from remote areas without SU availability, with the majority being transferred to hospitals with SUs.

The RFDS median overall transfer time of $238 \mathrm{~min}$ is also higher than what has been reported in other countries. A study from the Polish Medical Air Rescue found that the median time from activation to stroke patient transfer was $60 \mathrm{~min}$ [16]. Furthermore, a study from the Norwegian Air Ambulance Service indicated that it took 19 min flying time on average to reach PE patients [17]. However, the median flight time from mobilisation to scene (i.e., flight time to patient) by the RFDS was $58 \mathrm{~min}$ (as detailed in Fig. 2), which is similar to the Polish Medical Air Rescue transfer times. The longer median overall transfer time of the RFDS is most likely due to the sig- 
nificant longer distances the RFDS required to travel, due to the vastness of Australia, compared to other national retrieval services [7]. Fortunately, a prospective study is planned, in which response times among the leading aeromedical services of the world will be compared.

A precise stroke diagnosis is required to enable treatment. Without knowing the stroke type, ischaemic or haemorrhagic, through diagnostic imaging, acute reperfusion therapy cannot be instituted. Ischaemic strokes, caused by arteries being blocked or narrowed, require treatment to restore adequate blood flow to the brain, most often via intravenous $\mathrm{tPA}$. Even more dramatic benefits are seen with endovascular thrombectomy for patients with large artery occlusion $[13,18]$. Whereas haemorrhagic stroke, caused by extravasation of blood into the brain, requires measures to control the bleeding and reduce pressure in the brain $[1,18]$. However, this study demonstrated that the majority of aeromedical retrieval sites did not have access to diagnostic imaging, thus limiting stroke subtype diagnosis, immediate inflight treatment, and redirection to an appropriate comprehensive stroke centre.

The establishment of traditional SUs in rural and especially remote areas is not feasible due to sparse population distribution over vast areas. The greatest benefits in stroke intervention are achieved very early after onset, particularly in the first "golden hour" [19]. However, a major and persistent issue with the treatment of stroke in remote Australia is the vast geographical distance required to travel to access emergency care, significantly limiting intervention in the most critical time window [8]. As mentioned, we found that the majority of the remote population do not have access to radiology/imaging within 60 min drive time and that diagnostic imaging was not available for many of the aeromedical retrievals. This is consistent with an Australian report that found that patients living in rural and remote areas have "limited access to appropriate diagnostic imaging services; and patients face additional costs to access those services" [20]. This, coupled with remote emergency responders having limited access to up-to-date imaging facilities and specialist support, makes an appropriate diagnosis difficult [21].

This study needs to be put in the context of the revolution that has occurred in acute stroke therapies over the last few decades. Reperfusion therapies, in particular, have the potential to improve outcomes dramatically when administered early after the stroke onset. Means of delivering earlier prehospital therapies are emerging, for example, recent trials of prehospital models of early intervention with mobile stroke units (MSUs) have been im-

Aeromedical Retrieval for Stroke in Australia plemented in Australia [13, 22]. To date, these have been confined to metropolitan areas with high population density. These MSUs are specialised ambulances with onboard CT and point-of-care pathology tools with the potential for telemedicine connection to a stroke specialist. Early studies show that more patients can be treated with tPA and the time to treatment can be reduced by over 40 min $[14,19,23,24]$. Of interest, a Norwegian MSU in a similar rural setting has been able to provide stroke patients with thrombolysis within $60 \mathrm{~min}$ after the symptom onset. Similar to RFDS aeromedical retrievals, this service included an emergency physician [25]. How this emerging pre-hospital paradigm might be adapted to include an Australian rural and remote service provider or population needs to be addressed.

The RFDS already provides extensive aeromedical retrieval support to remote populations, and as such, there is a great potential for the RFDS to develop and equip an aircraft(s) to become an MSU [24]. Walter et al. [24] proposed that RFDS aircrafts, in addition to current medical equipment, could carry a specially designed CT scanner. However, there are two main issues with this proposal, including the weight of the CT machine and the optimal location of a single RFDS MSU. The issue of distances and the vast distribution of RFDS stroke patients make the provision of only a limited number of aeromedical MSUs impractical. An innovative and more expansive solution is needed.

For example, the potential of ultra-lightweight and low-cost brain imaging devices needs to be explored. For an RFDS patient receiving an aeromedical transfer for a stroke to a metropolitan hospital with an SU, the provision of a lightweight $\mathrm{CT}$ on the aircraft would potentially allow earlier stroke diagnosis and thrombolysis prior to transit. In addition to the RFDS, a lightweight and portable CT device could be deployed in several clinical settings, such as regional hospitals and road-based ambulance services, to make early stroke treatment a reality, independent of geographical location. To optimise the prehospital treatment of stroke, it is proposed that a transformative research program to develop, test, and implement novel disruptive technologies should be implemented.

This study was limited to RFDS patient data, and as such, it did not include other rural and remote healthcare providers. While all diagnoses were collected, a potential limitation is the accuracy of the recorded working diagnosis made in flight. However, it should be noted that this process is currently considered the gold standard in aeromedical medicine $[4,9]$. A further limitation was that we 
were unable to collect data from the time of onset of stroke symptoms to the transfer request. This limitation will be addressed in future studies, where all data will be prospectively accrued.

\section{Acknowledgements}

We would like to thank the supporters of the Royal Flying Doctor Service, who made this research project possible.

\section{Statement of Ethics}

This project was deemed a low-risk quality assurance project by the RFDS Clinical and Health Services Research Committee (CHSRC), which provides oversight for RFDS research projects, on 18 March 2019. As this project involved routinely collected data, specific patient consent forms were not required.

\section{Disclosure Statement}

The authors have no conflicts of interest to declare.

\section{Funding Sources}

The authors have no funding sources to declare.

\section{Author Contributions}

F.W.G. conceptualised the research, formulated the research design, and conducted data collection and analysis. L.B., A.D.S., D.E., F.Q., M.S., S.W., K.F., S.D., and G.A.D. helped conceptualise the research design and methods. P.S. assisted with data collection. L.C. and F.W.G. conducted data statistical analysis and presented the study results. All authors assisted in the final drafting of the manuscript. S.D. and G.A.D. contributed equally as the senior clinicians in the research team.

\section{References}

1 Zhai S, Gardiner F, Neeman T, Jones B, Gawarikar Y. The cost-effectiveness of a stroke unit in providing enhanced patient outcomes in an Australian teaching hospital. J Stroke Cerebrovasc Dis. 2017 Oct;26(10):2362-8.

2 Campbell BCV, De Silva DA, Macleod MR, Coutts SB, Schwamm LH, Davis SM, et al. Ischaemic stroke. Nat Rev Dis Primers. 2019 Oct 10;5(1):70.

3 Cadilhac DA, Kilkenny MF, Longworth M, Pollack MR, Levi CR. Metropolitan-rural divide for stroke outcomes: do stroke units make a difference? Intern Med J. 2011 Apr; 41(4):321-6.

4 Gardiner FW, Coleman M, Teoh N, Harwood A, Coffee NT, Gale L, et al. Aeromedical retrievals of people for mental health care and the low level of clinical support in rural and remote Australia. Med J Aust. 2019;211(8): 351-6.

5 Geoscience Australia. Area of Australia: states and territories. Canberra, Australia: Australian Government; 2020.

6 Australian Bureau of Statistics. Population estimates by age and sex, regions of Australia (ASGS 2016), 2018. Canberra, Australia: Australian Bureau of Statistics; 2018.

7 Brown WJ, Young AF, Byles JE. Tyranny of distance? The health of mid-age women living in five geographical areas of Australia. Aust J Rural Health. 1999;7(3):148-54.

8 Gardiner F, Gale L, Ransom A, Laverty M. Looking Ahead: responding to the health needs of country Australians in 2028- the centenary year of the RFDS. Canberra, Australia: The Royal Flying Doctor Service; 2018.

9 Gardiner FW, Bishop L, Gale L, Harwood A, Teoh N, Lucas RM, et al. Poor access to kidney disease management services in susceptible patient populations in rural Australia is associated with increased aeromedical retrievals for acute renal care. Intern Med J. 2019 Dec 10.

10 Stroke Foundation. National stroke Audit acute services report. Melbourne, Australia; 2017.

11 Tableau. Maps. Tableau; 2019.

12 Balabanski AH, Newbury J, Leyden JM, Arima $\mathrm{H}$, Anderson CS, Castle S, et al. Excess stroke incidence in young aboriginal people in South Australia: pooled results from two population-based studies. Int J Stroke. 2018 2018;13(8):811-4.

13 Campbell BC. Advances in stroke medicine. Med J Aust. 2019 May;212(1):46-e1.

14 Zhao H, Coote S, Langenberg F, Easton D, Bent L, Foster S, et al. Abstract 175: faster prehospital workflow in the Melbourne mobile stroke unit halves onset to reperfusion therapy. Stroke. 2019;50(Suppl_1):A175-A75.

15 Quain DA, Parsons MW, Loudfoot AR, Spratt NJ, Evans MK, Russell ML, et al. Improving access to acute stroke therapies: a controlled trial of organised pre-hospital and emergency care. Med J Aust. 2008 Oct 20;189(8):429-33.

16 Świeżewski SP, Rzońca P, Panczyk M, Leszczyński PK, Gujski M, Michalak G, et al. Polish helicopter emergency medical service (HEMS) response to stroke: a five-year retrospective study. Med Sci Monit. 2019;25:654753.

17 Zakariassen E, Uleberg O, Røislien J. Helicopter emergency medical services response times in Norway: do they matter? Air Med J. 2015;34(2):98-103.

18 Goyal M, Menon BK, van Zwam WH, Dippel DW, Mitchell PJ, Demchuk AM, et al. Endovascular thrombectomy after large-vessel ischaemic stroke: a meta-analysis of individual patient data from five randomised trials. Lancet. 2016 Apr 23;387(10029):1723-31.

19 Ebinger M, Kunz A, Wendt M, Rozanski M, Winter B, Waldschmidt C, et al. Effects of golden hour thrombolysis: a prehospital acute neurological treatment and optimization of medical care in stroke (PHANTOM-S) substudy. JAMA Neurol. 2015 Jan;72(1):25-30.

20 Commonwealth of Australia. Availability and accessibility of diagnostic imaging equipment around Australia. Canberra, Australia: Senate Standing Committees on Community Affairs; 2018.

21 Lyon M, Blaivas M, Brannam L. Use of emergency ultrasound in a rural ED with limited radiology services. Am J Emerg Med. 2005 Mar;23(2):212-4.

22 Coote S, Zhao H, Pesavento L, Langenberg F, Desmond P, Easton D, et al. Abstract TP291: Melbourne mobile stroke unit increases stroke treatment rates and treatment opportunities. Stroke. 2019;50(Suppl 1):ATP291ATP91.

23 Rajan SS, Rajan S, Baraniuk S, Parker S, Wu TC, Bowry R, et al. Implementing a mobile stroke unit program in the United States: why, how, and how much? JAMA Neurol. 2015 Feb;72(2):229-34.

24 Walter S, Zhao H, Easton D, Bil C, Sauer J, Liu $\mathrm{Y}$, et al. Air-mobile stroke unit for access to stroke treatment in rural regions. Int J Stroke. 2018 Aug;13(6):568-75.

25 Larsen K, Bache KG, Franer E, Tveit LH, Hov MR, Lund CG, et al. Pre-hospital thrombolysis of ischemic stroke in the emergency service system-a case report from the treat-NASPP trial. Acta Anaesthesiol Scand. 2019;63(3): 410-3. 